QUARTERLY OF APPLIED MATHEMATICS

VOLUME LXIV, NUMBER 3

SEPTEMBER 2006, PAGES 515-527

S $0033-569 X(06) 01014-5$

Article electronically published on August 9, 2006

\title{
THE LAMBERT TRANSFORM FOR SMALL AND LARGE VALUES OF THE TRANSFORMATION PARAMETER
}

\author{
BY \\ CHELO FERREIRA (Departamento de Matemática Aplicada, Universidad de Zaragoza, Spain) \\ AND \\ JOSÉ L. LÓPEZ (Departamento de Matemática e Informática, Universidad Pública de Navarra, \\ Spain)
}

Abstract. We derive asymptotic expansions of the Lambert transform

$$
\int_{0}^{\infty} x t\left(e^{x t}-1\right)^{-1} f(t) d t
$$

of a locally integrable function $f(t)$ for small and large $x$. All the expansions are accompanied by error bounds for the remainder at any order of the approximation.

1. Introduction. Any series of the form

$$
\sum_{n=1}^{\infty} a_{n} \frac{x^{n}}{1-x^{n}}, \quad \quad|x|<1, \quad a_{n} \in \mathbf{C} .
$$

is called a Lambert series. It is a natural generalization of the following formulas related to the theory of numbers and proved by Lambert [18]:

$$
\sum_{n=1}^{\infty} n \frac{x^{n}}{1-x^{n}}=\sum_{n=1}^{\infty} \sigma(n) x^{n}, \quad \sum_{n=1}^{\infty} \frac{x^{n}}{1-x^{n}}=\sum_{n=1}^{\infty} \tau(n) x^{n}, \quad|x|<1,
$$

where $\sigma(n)$ and $\tau(n)$ represent the sum and the number of the divisors of $n$ respectively.

In the same way as the Laplace integral is a generalization of the power series, Widder introduced the Lambert transform as a generalization of the Lambert series (1.1). The Lambert transform of a function $f(t)$ is defined by [16]

$$
L(x) \equiv \int_{0}^{\infty} \frac{x t}{e^{x t}-1} f(t) d t, \quad x \in \mathbf{C},
$$

when the integral exists. In the remainder of the paper we consider $\Re x>0, f(t) \in$ $L_{\mathrm{Loc}}^{1}[0, \infty)$ and $|f(t)| \leq M e^{x_{0} t}$ for $t \geq T$ and certain $x_{0}<\Re x, M>0$ and $T>0$.

Received November 28, 2005.

2000 Mathematics Subject Classification. Primary 41A60, 65R10; Secondary 33B15.

Key words and phrases. Lambert transform, asymptotic expansions, error bounds.

E-mail address: cferrei@unizar.es

E-mail address: j1.lopez@unavarra.es

(C)2006 Brown University 
The Lambert transform appears in the computation of the observable radiation $(L(x))$ in terms of the distribution of radiation $(f(t))$ of black bodies at different temperatures $t$ 14.

Several properties of the Lambert transform and inversion formulas have been studied by different authors. Among other results, we remark the following. Miller studied convergence properties of $L(x)$ needed to develop inversion formulas [8] and introduced summability techniques for power series which use the Lambert transform [7]. Widder derives an inversion formula in terms of a limit of the derivatives of $L(x)$ which involves the Möbius function [16. Different inversion formulae related to the inversion formula given by Widder are studied in [9], [11] and [15]. Raina and Srivastava introduced a generalization of the Lambert transform related with the generalized Riemann zeta function [13. In [5] the authors give the inversion formula for this generalized Lambert transform. Raina and Nahar introduced a generalization of the Lambert transform related with a class of functions connected to the Hurwitz zeta function [12. Goldberg introduced a more general kernel for the Lambert transform working with transforms as Stieltjes integrals and derived some inversion formulae [4. More recently, Hayek et al. have studied this generalized Lambert transform on the space of distributions of compact support giving an inversion formula over this space [6].

On the other hand, complete asymptotic expansions including error bounds of $L(x)$ for large and small $x$ are not fully investigated. The purpose of this paper is to obtain asymptotic expansions of $L(x)$ for large and small values of the variable $x$. We face the challenge of obtaining easy algorithms to compute the coefficients of these expansions as well as error bounds at any order of the approximation.

We will apply the ideas of Watson's lemma to obtain asymptotic expansions of $L(x)$ for large $x$ and also for small $x$ when the positive moments of $f(t)$ exist. When those moments do not exist, we will use an alternative approach based on the theory of distributions introduced by McClure and Wong [17], chap. 6] to derive asymptotic expansions of $L(x)$ for small $x$.

In section 2 we derive an asymptotic expansion of (1.2) for large $x$ (Theorem 2.1) and three asymptotic expansions for small $x$ (Theorems 2.2-2.4) accompanied by error bounds. Several numerical examples are shown in section 3.

2. Asymptotic expansions. An asymptotic expansion of $L(x)$ for large $x$ is obtained in the following theorem by using the idea of Watson's lemma. To this end we require for $f(t)$ an asymptotic expansion at $t=0$ :

$$
f(t)=\sum_{k=0}^{n-1} a_{k} t^{k+\alpha}+r_{n}(t), \quad r_{n}(t)=\mathcal{O}\left(t^{n+\alpha}\right) \text { when } t \rightarrow 0^{+},
$$

where $\alpha>-1$ and $a_{k} \in \mathbf{C}$.

Theorem 2.1. Let $f(t)$ satisfy (2.1). Then, for $|\operatorname{Arg}(x)|<\pi / 2$, and $n=1,2,3, \ldots$,

$$
\int_{0}^{\infty} \frac{x t}{e^{x t}-1} f(t) d t=\sum_{k=0}^{n-1} \Gamma(k+\alpha+2) \zeta(k+\alpha+2) \frac{a_{k}}{x^{k+\alpha+1}}+R_{n}(x),
$$


where $\zeta(z)$ denotes the zeta function. The remainder term is defined by

$$
R_{n}(x) \equiv \int_{0}^{\infty} \frac{x t}{e^{x t}-1} r_{n}(t) d t
$$

where $r_{n}(t)$ is given in (2.1), and verifies $R_{n}(x)=\mathcal{O}\left(x^{-n-1-\alpha}\right)$ when $|x| \rightarrow \infty$.

Proof. Introducing the expansion (2.1) into (1.2) and interchanging the sum and the integral we obtain

$$
L(x)=x \sum_{k=0}^{n-1} a_{k} \int_{0}^{\infty} \frac{t^{k+\alpha+1} d t}{e^{x t}-1}+x \int_{0}^{\infty} \frac{t r_{n}(t)}{e^{x t}-1} d t .
$$

Performing the change of variable $x t \rightarrow t$ in the first integral above and using Cauchy's residue theorem and [1], eq. 23.2.7] we obtain (2.2) $-(2.3)$.

From (2.1), $\exists t_{0}>0$ such that $\left|r_{n}(t)\right| \leq c_{n} t^{n+\alpha}$ for $0 \leq t \leq t_{0}$ and some positive constant $c_{n}$. On the other hand, from (2.1) and from the paragraph following (1.2) we have that $\left|r_{n}(t)\right| \leq M e^{x_{0} t}+\sum_{k=0}^{n-1}\left|a_{k}\right| t^{k+\alpha}$ for $t \geq T$ with $x_{0}<\Re x$. Therefore,

$$
\begin{aligned}
& \frac{\left|R_{n}(x)\right|}{|x|} \leq \int_{0}^{t_{0}} \frac{t\left|r_{n}(t)\right|}{e^{t \Re x}-1} d t+\int_{t_{0}}^{T} \frac{t\left|r_{n}(t)\right|}{e^{t \Re x}-1} d t+\int_{T}^{\infty} \frac{t\left|r_{n}(t)\right|}{e^{t \Re x}-1} d t \\
& \leq c_{n} \int_{0}^{t_{0}} \frac{t^{n+1+\alpha}}{e^{t \Re x}-1} d t+\int_{t_{0}}^{T} \frac{t\left|r_{n}(t)\right|}{e^{t_{0} \Re x}-1} d t+\int_{T}^{\infty} \frac{M e^{x_{0} t}+\sum_{k=0}^{n-1}\left|a_{k}\right| t^{k+\alpha}}{e^{t \Re x}-1} d t \\
& \leq \frac{c_{n}}{(\Re x)^{n+\alpha+2}} \int_{0}^{\infty} \frac{t^{n+1+\alpha}}{e^{t}-1} d t+\left(e^{t_{0} \Re x}-1\right)^{-1} \int_{t_{0}}^{T} t\left|r_{n}(t)\right| d t \\
&+e^{\left(x_{0}-\Re x\right) T} \int_{0}^{\infty} \frac{(t+T)}{e^{t \Re x}-e^{-T \Re x}}\left[M e^{x_{0} t}+e^{-x_{0} T} \sum_{k=0}^{n-1}\left|a_{k}\right|(t+T)^{k+\alpha}\right] d t .
\end{aligned}
$$

After some examination of the two last lines above we see that $R_{n}(x)=\mathcal{O}\left((\Re x)^{-n-1-\alpha}\right)$ and then, (2.2) is an asymptotic expansion for large $x$.

The bound obtained above for $R_{n}(x)$ is not useful for numerical computations. But if the bound $\left|r_{n}(t)\right| \leq c_{n} t^{n+\alpha}$ holds $\forall t \geq 0$ and not only for $0 \leq t \leq t_{0}$, a more precise bound may be obtained. This is the subject of the following proposition.

Proposition 2.1. Suppose that the remainder $r_{n}(t)$ in the expansion (2.1) satisfies the bound $\left|r_{n}(t)\right|<c_{n} t^{n+\alpha} \forall t \in[0, \infty)$ for some positive constant $c_{n}$. Then:

i) An error bound for the remainder in (2.2) is given by

$$
\left|R_{n}(x)\right| \leq c_{n} \Gamma(n+\alpha+2) \zeta(n+\alpha+2) \frac{|x|}{(\Re x)^{n+\alpha+2}} .
$$

ii) If the expansion (2.1) verifies the error test, we can take $c_{n}=\left|a_{n}\right|$ in the above formula.

iii) If $f(w)$ is a bounded analytic function in the region $W_{0}$ of the complex $z$-plane comprised by the points situated at a distance $\leq r$ from the positive real axis (see Fig. 1(a)), we can take $c_{n}=C r^{-n-\alpha}$ and $C$ a bound of $f(w)$ in $W_{0}$. 


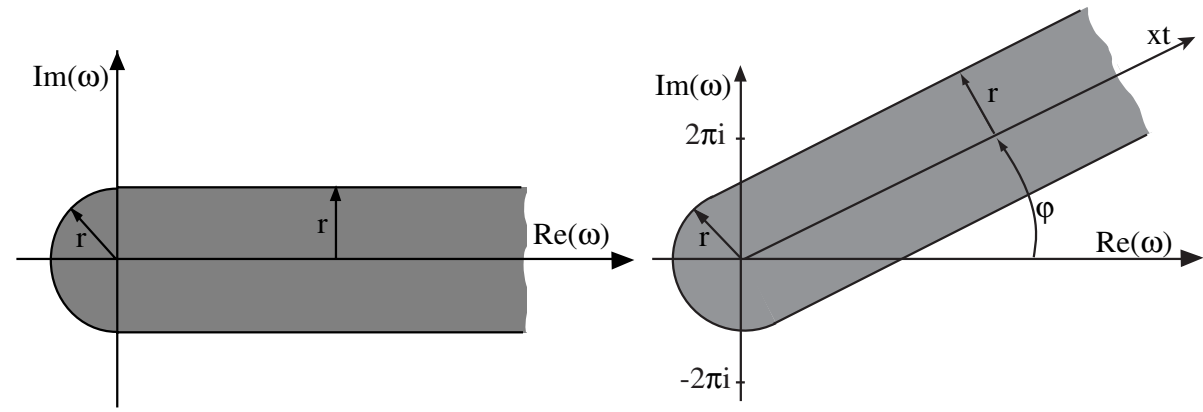

Fig. 1. (a) Region $W_{0}$ considered in (iii) in Proposition 2.1. (b) Region $W$ considered in Theorem 2.2 with $\varphi=\operatorname{Arg}(x),|\operatorname{Arg}(x)|<$ $\pi / 2$. The larger $\varphi$ is, the smaller $r$ is in order to assure that $\pm 2 \pi i$ is out of $W$.

Proof. From (2.3) we have

$$
\left|R_{n}(x)\right| \leq|x| \int_{0}^{\infty} \frac{\left|r_{n}(t)\right| t d t}{e^{t \Re x}-1} .
$$

Introducing the bound $\left|r_{n}(t)\right|<c_{n} t^{n+\alpha}$ and after straightforward computations we obtain (2.4).

Thesis (ii) is proved in [[10, p. 68]. Thesis (iii) is proved in [3].

In the following three theorems we derive three asymptotic expansions of $L(x)$ for small $x$ depending on whether the positive moments of $f(t)$ exist or not.

Theorem 2.2. Let $f(t)$ satisfy $t^{n} f(t) \in \mathcal{L}^{1}[0, \infty)$ for $n=0,1, \ldots$. Then, for $\Re x>0$,

$$
\int_{0}^{\infty} \frac{x t}{e^{x t}-1} f(t) d t=\sum_{k=0}^{n-1} \frac{B_{k}}{k !} M[f ; k+1] x^{k}+R_{n}(x), \quad n=1,2,3, \ldots,
$$

where $B_{n}$ are the Bernoulli numbers and $M[f ; k]$ denotes the Mellin transform of $f(t)$ : $\int_{0}^{\infty} t^{k-1} f(t) d t$. The remainder $R_{n}(x)$ verifies

$$
\left|R_{n}(x)\right| \leq C \frac{M[|f| ; n+1]}{[\pi \cos (\operatorname{Arg}(x))]^{n}}|x|^{n},
$$

where $C$ is a bound of $\left|w /\left(e^{w}-1\right)\right|$ in the region $W$ comprised by the points situated at a distance $\leq r$ from the straight line $w=t e^{i \operatorname{Arg}(x)}, t \in[0, \infty)$ for any $r>0$ such that $\pm 2 \pi i \notin W$ (see Fig. 1(b)). A possible value for $C$ is:

$$
\begin{aligned}
C=\operatorname{Max}_{x_{0}, x_{1}}\left\{\frac{\sqrt{x_{0}^{2}+\pi^{2} \cos ^{2}(\operatorname{Arg}(x)) \pm 2 x_{0} \pi \sin (\operatorname{Arg}(x)) \cos (\operatorname{Arg}(x))}}{\sin \left(x_{0} \tan (\operatorname{Arg}(x))\right)},\right. \\
\left.\frac{\pi \cos (\operatorname{Arg}(x))}{\sqrt{e^{2 x_{1}}+1-2 e^{x_{1}} \cos \left(\sqrt{\pi^{2} \cos ^{2}(\operatorname{Arg}(x))-x_{1}^{2}}\right)}}\right\},
\end{aligned}
$$


where $x_{0}$ are the solutions of the equation

$$
\tan (\operatorname{Arg}(x)) \sin (x \tan (\operatorname{Arg}(x)))=e^{x}+\cos (x \tan (\operatorname{Arg}(x)))
$$

in the interval $[-\pi|\sin (\operatorname{Arg}(x))| \cos (\operatorname{Arg}(x)), \infty)$ and $x_{1}$ are the solutions of the equations

$x \sin \sqrt{\pi^{2} \cos ^{2}(\operatorname{Arg}(x))-x^{2}}= \pm\left(e^{x}-\cos \sqrt{\pi^{2} \cos ^{2}(\operatorname{Arg}(x))-x^{2}}\right) \sqrt{\pi^{2} \cos ^{2}(\operatorname{Arg}(x))-x^{2}}$

in the respective intervals $[-\pi \cos (\operatorname{Arg}(x)), \mp \pi \sin (\operatorname{Arg}(x)) \cos (\operatorname{Arg}(x)))$.

Proof. From [1], eq. 23.1.1] we have

$$
\frac{x t}{e^{x t}-1}=\sum_{k=0}^{n-1} \frac{B_{k}}{k !}(x t)^{k}+r_{n}(x t), \quad n=1,2,3,5,7,9, \ldots,
$$

where $r_{n}(x t)=\mathcal{O}\left((x t)^{n}\right)$ when $x t \rightarrow 0$. Introducing this expansion in (1.2) and replacing the sum and the integral we obtain (2.5) with

$$
R_{n}(x) \equiv \int_{0}^{\infty} r_{n}(x t) f(t) d t
$$

By using Cauchy's integral formula for the derivative of an analytic function in the Lagrange form for the remainder $r_{n}(x t)$ in the Taylor expansion (2.8) we obtain [2]:

$$
\left|r_{n}(x t)\right| \leq \frac{C t^{n}|x|^{n}}{[\pi \cos (\operatorname{Arg}(x))]^{n}}, \quad n=1,2,3, \ldots
$$

Introducing this bound in (2.9) we obtain (2.6), which shows the asymptotic character of the expansion (2.5). Formula (2.7) is taken from [2, Eq. 16].

The following proposition offers a more accurate error bound than (2.6) when $x>0$.

Proposition 2.2. For $x>0$, an error bound for the remainder in (2.5) is given by

$$
\left|R_{n}(x)\right| \leq\left\{\frac{\left|B_{n}\right|}{n !} \int_{0}^{2 \pi /|x|} t^{n}|f(t)| d t+\frac{\left(1-e^{-\pi}\right)^{-1}}{\pi^{n-1}} \int_{2 \pi /|x|}^{\infty} t^{n}|f(t)| d t\right\} x^{n} .
$$

Proof. For $0 \leq x<2 \pi$, (2.8) is a convergent expansion where two consecutive error terms have opposite signs [2]. Then, applying the error test ([10], p. 68) we find

$$
\left|r_{n}(x t)\right| \leq \frac{\left|B_{n}\right|}{n !}(x t)^{n}, \quad 0 \leq x t<2 \pi, \quad n=1,2,3, \ldots .
$$

From (2.9) we have

$$
\left|R_{n}(x)\right| \leq \int_{0}^{2 \pi / x}\left|r_{n}(x t)\right||f(t)| d t+\int_{2 \pi / x}^{\infty}\left|r_{n}(x t)\right||f(t)| d t .
$$

Now we introduce the bound (2.12) in the first integral and the bound (2.10), with $C(0)=\pi /\left(1-e^{-\pi}\right)$, in the second one. From here, (2.11) follows after straightforward manipulations.

The previous theorem does not work when the positive moments of $f(t)$ do not exist. In this case we can use the distributional technique of McClure and Wong 
[[17, chap. 6]. To apply this technique we require for $f(t)$ an asymptotic expansion at infinity:

$$
f(t)=\sum_{k=K}^{n-1} \frac{a_{k}}{t^{k+\alpha}}+f_{n}(t), \quad f_{n}(t)=\mathcal{O}\left(t^{-n-\alpha}\right), \quad \text { when } \quad t \rightarrow \infty,
$$

where $K \in \mathbf{Z}, 0<\alpha \leq 1$ and $a_{k} \in \mathbf{C}$.

Theorem 2.3. Let $f(t)$ satisfy (2.13) with $0<\alpha<1$. Then, for $\Re x>0$,

$$
\begin{aligned}
\int_{0}^{\infty} \frac{x t}{e^{x t}-1} f(t) d t= & \sum_{k=K}^{n-1} a_{k} \Gamma(2-\alpha-k) \zeta(2-\alpha-k) x^{k+\alpha-1} \\
& +\sum_{k=0}^{n-1} \frac{B_{k}}{k !} M[f ; k+1] x^{k}+R_{n}(x),
\end{aligned}
$$

where

$$
\begin{gathered}
R_{n}(x) \equiv(-1)^{n} \int_{0}^{\infty} f_{n, n}(t) \frac{d^{n}}{d t^{n}}\left(\frac{x t}{e^{x t}-1}\right) d t \\
f_{n, n}(t) \equiv \frac{(-1)^{n}}{(n-1) !} \int_{t}^{\infty}(u-t)^{n-1} f_{n}(u) d u
\end{gathered}
$$

In (2.14),$M[f ; k+1]$ denotes the Mellin transform of $f(t)$ at $z=k+1, \int_{0}^{\infty} t^{z-1} f(t) d t$, or its analytic continuation at that point.

Proof. Consider the tempered distributions $\mathbf{f}, \mathbf{t}_{+}^{-k-\alpha}$ and $\mathbf{f}_{n}$ associated to the corresponding functions $f(t), t^{-k-\alpha}$ and $f_{n}(t)$ which appear in (2.13). They act over functions $h(t) \in \mathcal{S}$ (the Schwarz class of $\mathcal{C}^{(\infty)}$ rapidly decreasing functions) in the following way [17], chap. 6]:

$$
\begin{array}{ll}
\langle\mathbf{f}, h\rangle=\quad \int_{0}^{\infty} f(t) h(t) d t, \quad\left\langle\mathbf{f}_{n}, h\right\rangle=(-1)^{n} \int_{0}^{\infty} f_{n, n}(t) h^{(n)}(t) d t \\
\left\langle\mathbf{t}_{+}^{\mathbf{k}-\alpha}, h\right\rangle=\int_{0}^{\infty} t^{k-\alpha} h(t) d t, & \left\langle\mathbf{t}_{+}^{-k-\alpha}, h\right\rangle=\frac{1}{(\alpha)_{k}} \int_{0}^{\infty} t^{-\alpha} h^{(k)}(t) d t
\end{array}
$$

for $k=0,1,2, \ldots$ From [[17], chap. 6, lemma 1] we have that these distributions are related by the equality:

$$
\mathbf{f}=\sum_{k=K}^{n-1} a_{k} \mathbf{t}_{+}^{-k-\alpha}+\sum_{k=0}^{n-1} \frac{(-1)^{k}}{k !} M[f ; k+1] \delta^{(\mathbf{k})}+\mathbf{f}_{n},
$$

where $\delta^{(k)}$ is the $k$-th derivative of the delta distribution at the origin: $\left\langle\delta^{(k)}, h\right\rangle=$ $(-1)^{k} h^{(k)}(0)$. Here, $M[f ; k+1]$ denotes the Mellin transform of $f(t)$ at $z=k$ or its analytic continuation. The third integral in (2.17) is indeed the Mellin transform of $h(t)$, $M[h ; z]$, at $z=k+1-\alpha:\left\langle\mathbf{t}_{+}^{\mathbf{k}-\alpha}, h\right\rangle=M[h ; k+1-\alpha]$. Moreover, by integrating by parts it may be proved that the last integral in (2.17) is the analytic continuation of $M[h ; z]$ to the point $z=-k+1-\alpha$. Therefore, applying (2.18) to the function $h(t)=x t /\left(e^{x t}-1\right)$ and using (2.17) and [1], eq. 23.1.1; eq. 23.2.7] we obtain (2.14)-(2.15). 
Theorem 2.4. Let $f(t)$ satisfy (2.13) with $\alpha=1$. Then, for $\Re x>0$,

$$
\begin{aligned}
\int_{0}^{\infty} \frac{x t}{e^{x t}-1} f(t) d t & =\sum_{k=K}^{-1} \Gamma(1-k) \zeta(1-k) x^{k}-\log x+\sum_{k=1}^{n-1} a_{k}\left[\frac { ( - 1 ) ^ { k - 1 } } { ( k - 1 ) ! } \left(\zeta^{\prime}(-k+1)\right.\right. \\
& \left.-\log x \zeta(-k+1))-\frac{B_{k}}{k !}\left(\gamma+\frac{1}{k}\right)\right] x^{k}+\sum_{k=0}^{n-1} \frac{B_{k}}{k !} c_{k+1} x^{k}+R_{n}(x),
\end{aligned}
$$

where

$$
c_{n+1} \equiv \lim _{z \rightarrow n}\left[M[f ; z+1]+\frac{a_{n}}{z-n}\right]+a_{n}(\gamma+\psi(n+1)),
$$

$\gamma$ is the Euler constant and $\psi$ the digamma function. $R_{n}(x)$ is given in (2.15)-(2.16).

Proof. It is similar to the proof of Theorem 2.3. But now, the second line of (2.17) is replaced by

$$
\left\langle\mathbf{t}_{+}^{k}, h\right\rangle=\int_{0}^{\infty} t^{k} h(t) d t, \quad\left\langle\mathbf{t}_{+}^{-\mathbf{k}-\mathbf{1}}, h\right\rangle=-\frac{1}{k !} \int_{0}^{\infty} h^{(k+1)}(t) \log t d t
$$

for $k=0,1,2, \ldots$. As in the preceding proof, $\left\langle\mathbf{t}_{+}^{\mathbf{k}}, h\right\rangle=M[h ; k+1]$. But now, $\left\langle\mathbf{t}_{+}^{-k-1}, h\right\rangle$ is related to $M[h ;-k]$ by means of a more sophisticated formula:

$$
\left\langle\mathbf{t}_{+}^{-k-1}, h\right\rangle=\lim _{z \rightarrow-k}\left[M[h ; z]-\frac{h^{k}(0)}{k !(z+k)}\right]-\frac{h^{k}(0)}{k !}(\psi(k+1)+\gamma) .
$$

In this formula, $M[h ; z]$ represents indeed the analytic continuation of $\int_{0}^{\infty} t^{z-1} h(t) d t$ and may be derived by means of integration by parts. Using this last formula and the first formula of (2.21) for $h(t)=x t /\left(e^{x t}-1\right)$ we obtain [1], eq. 23.2.5]:

$$
\left\{\begin{array}{l}
x^{k} \Gamma(1-k) \zeta(1-k) \quad \text { if } \quad k<0, \\
-\log x \text { if } k=0, \\
x^{k}\left[\frac{(-1)^{k-1}}{(k-1) !}\left(\zeta^{\prime}(-k+1)-\zeta(-k+1) \log x\right)-\frac{B_{k}}{k !}\left(\gamma+\frac{1}{k}\right)\right] \quad \text { if } \quad k>0 .
\end{array}\right.
$$

To complete this proof, formula (2.18) must also be replaced by [[17], chap. 6, lemma 2]:

$$
\mathbf{f}=\sum_{k=K}^{n-1} a_{k} \mathbf{t}_{+}^{-k-1}+\sum_{k=0}^{n-1} \frac{(-1)^{k}}{k !} c_{k+1} \delta^{(k)}+\mathbf{f}_{\mathbf{n}},
$$

where $c_{k}$ are given in (2.20). Applying this formula to the function $h(t)=x t /\left(e^{x t}-1\right)$ and using the first line in (2.17) and (2.22) we obtain (2.19) $-(2.20)$.

Until this moment, the expansions (2.14) and (2.19) are formal asymptotic expansions for small $x$. In the following theorem we show that those expansions are indeed asymptotic expansions.

Theorem 2.5. For $\Re x>0$, the remainder term $R_{n}(x)$ in Theorems 2.3 and 2.4 satisfies:

$$
\begin{array}{cc}
\left|R_{n}(x)\right| \leq C_{n}|x|^{n+\alpha-1} & \text { if } 0<\alpha<1 \quad \text { and } \\
\left|R_{n}(x)\right| \leq C_{n}|x|^{n}|\log x| & \text { if } \alpha=1,
\end{array}
$$

where $C_{n}$ is a positive constant independent of $|x|$. 
Proof. From [[3], theorem 5] we have that $\left|f_{n, n}(t)\right| \leq C_{1, n} t^{-\alpha} \forall t \in[0, \infty)$ and $0<$ $\alpha<1$, where $C_{1, n}$ is a certain positive constant. On the other hand we write

$$
\frac{x t}{e^{x t}-1}=e^{-x t / 2} \phi(x t), \quad \phi(t) \equiv \frac{t e^{-t / 2}}{1-e^{-t}} .
$$

Then,

$$
\left|\frac{d^{n}}{d t^{n}}\left(\frac{x t}{e^{x t}-1}\right)\right| \leq e^{-t \Re x / 2}|x|^{n} \sum_{j=0}^{n}\left(\begin{array}{l}
n \\
j
\end{array}\right) 2^{-j}\left|\phi^{(n-j)}(x t)\right| .
$$

Using the Cauchy formula for the derivative of an analytic function we obtain

$$
\left|\phi^{(n-j)}(x t)\right| \leq C \frac{(n-j) !}{r^{n-j}}
$$

where $0<r<2 \pi$ and $C$ is a bound for $\phi(w)$ in its analyticity region $W$ given in Fig. 1 (b) with $\varphi=\operatorname{Arg}(x)$. Introducing this bound in (2.25) we have

$$
\left|\frac{d^{n}}{d t^{n}}\left(\frac{x t}{e^{x t}-1}\right)\right| \leq \frac{C|x|^{n}}{e^{t \Re x / 2}} \sum_{j=0}^{n}\left(\begin{array}{l}
n \\
j
\end{array}\right) \frac{(n-j) !}{2^{j} r^{n-j}}=\Gamma(n+1, r / 2) \frac{e^{r / 2}}{r^{n}} \frac{C|x|^{n}}{e^{t \Re x / 2}} .
$$

Introducing the bound $\left|f_{n, n}(t)\right| \leq C_{1, n} t^{-\alpha}$ and the bound (2.26) in (2.15) we obtain (2.23).

On the other hand, for $\alpha=1$ we have [[3], theorem 5] $\left|f_{n, n}(t)\right| \leq C_{2, n} t^{-1} \forall t \in\left[t_{0}, \infty\right)$, and $\left|f_{n, n}(t)\right| \leq C_{3, n}(|\log (t)|+1) \forall t \in\left[0, t_{0}\right]$, where $t_{0}, C_{2, n}$ and $C_{3, n}$ are certain positive constants. Dividing the integration interval of (2.15) at $t=t_{0}$ and using these bounds and (2.26) we obtain the bound (2.24).

The bounds given in Theorem 2.5 are not useful for numerical computations unless we are able to calculate the constants $C_{n}$ in terms of the dates of the problem. The following two propositions show that, if the bound $\left|f_{n}(t)\right| \leq c_{n} t^{-n-\alpha}$ holds $\forall t \in[0, \infty)$ and not only for $t \in\left[t_{0}, \infty\right)$, the constants $C_{n}$ in Theorem 2.5 can be calculated in terms of the constant $c_{n}$.

Proposition 2.3. Suppose that the remainder $f_{n}(t)$ in the expansion (2.13) satisfies the bound $\left|f_{n}(t)\right| \leq c_{n} t^{-n-\alpha} \forall t \in(0, \infty)$ for some positive constant $c_{n}$. Then,

(i) The remainder $R_{n}(x)$ in the expansion (2.14) satisfies

$$
\left|R_{n}(x)\right| \leq \frac{c_{n} C \pi 2^{1-\alpha}}{\sin (\pi \alpha) \Gamma(n+\alpha)} \frac{e^{r / 2} \Gamma(n+1, r / 2)}{r^{n} \cos ^{1-\alpha}(\operatorname{Arg}(x))}|x|^{n+\alpha-1},
$$

where $0<r<2 \pi$ and $C$ is a bound for $\phi(w)=w e^{-w / 2} /\left(1-e^{-w}\right)$ in its analyticity region $W$ given in Fig. 1(b) with $\varphi=\operatorname{Arg}(x)$.

(ii) If the expansion (2.13) verifies the error test, we can take $c_{n}=\left|a_{n}\right|$ in the above formula.

(iii) If $f(w)$ is a bounded analytic function in the region $W_{0}$ of the complex $z$-plane comprised of the points situated at a distance $\leq \tilde{r}$ from the positive real axis (see Fig. 1(a)), we can take $c_{n}=\tilde{C} \tilde{r}^{-n-\alpha}$ and $\tilde{C}$ a bound of $f(w)$ in $W_{0}$. 
Proof. Introducing the bound $\left|f_{n}(t)\right| \leq c_{n} t^{-n-\alpha}$ in the definition (2.16) of $f_{n, n}(t)$ we obtain

$$
\left|f_{n, n}(t)\right| \leq \frac{c_{n} \Gamma(\alpha)}{\Gamma(n+\alpha)} \frac{1}{t^{\alpha}} \quad \forall t \in[0, \infty) .
$$

Introducing (2.26) and (2.28) in (2.15), we obtain (2.27). The proof of (ii) and (iii) is as in Proposition 2.1.

Proposition 2.4. Suppose that each remainder $f_{n}(t)$ in the expansion (2.13) satisfies the bound $\left|f_{n}(t)\right| \leq c_{n} t^{-n-1}, \forall t \in(0, \infty)$ for some positive constant $c_{n}$. Then,

(i) The remainder $R_{n}(x)$ in the expansion (2.19) satisfies the bound

$$
\left|R_{n}(x)\right| \leq \frac{\bar{c}_{n} C \pi \sqrt{2}}{\Gamma(n+1 / 2)} \Gamma(n+1, r / 2) \frac{e^{r / 2}}{r^{n}}|x|^{n-1 / 2},
$$

where $\bar{c}_{n} \equiv \operatorname{Max}\left\{c_{n}, c_{n-1}+\left|a_{n-1}\right|\right\}, 0<r<2 \pi$ and $C$ is a bound for $\phi(w)$ in its analyticity region $W$ given in Fig. 1 (b) with $\varphi=\operatorname{Arg}(x)$. It also satisfies the bound

$\left|R_{n}(x)\right| \leq C \Gamma(n+1, r / 2) \frac{e^{r / 2}}{r^{n}} \frac{\left(c_{n-1}+\left|a_{n-1}\right|\right) \varepsilon+c_{n}+\frac{c_{n}}{n} \Theta(x, \varepsilon)}{(n-1) !}|x|^{n}$,

where $\varepsilon$ is an arbitrary positive number, and

$$
\Theta(x, \varepsilon) \equiv\left\{\begin{array}{cl}
2 e^{-1 / 2}-\log (\varepsilon|x|) & \text { if } \quad \varepsilon|x|<1, \\
\frac{2 e^{-\varepsilon|x| / 2}}{\varepsilon|x|} & \text { if } \quad \varepsilon|x| \geq 1
\end{array}\right.
$$

For small enough $x$ and fixed $n$, the optimum value for $\varepsilon$ is given by

$$
\varepsilon=\frac{c_{n}}{n\left(c_{n-1}+\left|a_{n-1}\right|\right)}
$$

(ii) If the expansion (2.13) verifies the error test, we can take $c_{n}=\left|a_{n}\right|$ in the above formula.

(iii) If $f(w)$ is a bounded analytic function in the region $W_{0}$ of the complex $z$-plane comprised of the points situated at a distance $\leq \tilde{r}$ from the positive real axis (see Fig. 1(a)), we can take $c_{n}=\tilde{C} \tilde{r}^{-n-\alpha}, \tilde{C}$ a bound of $f(w)$ in $W_{0}$.

Proof. From [ [3, proposition 2] we have

$$
\left|f_{n, n}(t)\right| \leq \frac{1}{(n-1) !}\left[\left(c_{n-1}+\left|a_{n-1}\right|\right) \log \left(\frac{\varepsilon}{t}\right)+\frac{c_{n}}{\varepsilon}\right] \quad \forall t \in(0, \varepsilon],
$$

where $\varepsilon>0$ is a fixed point, and

$$
\left|f_{n, n}(t)\right| \leq \frac{c_{n}}{n !} \frac{1}{t} \quad \forall t \in(0, \infty) .
$$

We divide the integration interval in the right-hand side of (2.15) at the point $t=\varepsilon$. On the one hand we use the bound (2.34) for $f_{n, n}(t)$ in the integral over $[\varepsilon, \infty)$ and the bound (2.33) in the integral over $(0, \varepsilon]$. On the other hand we use again (2.26). Then, we obtain (2.30)-(2.31). For small $x$ and fixed $n$, this bound takes its optimum value for $\epsilon$ given in (2.32). 
For deriving (2.29), we use that $f_{n}(t)$ satisfies the bound required in Proposition 2.1 with $\alpha=1 / 2$ and $c_{n}$ replaced by $\bar{c}_{n}$ [[3], proposition 2].

3. Numerical experiments. Tables 1-7 show numerical experiments on the accuracy of the approximations and error bounds supplied by Theorems 2.1-2.5 and Propositions 2.1-2.4.

In all these tables, the second column represents the value of $L(x)$ or $L(x) / x$. The third and sixth columns represent, respectively, a first (or a second) and a second (or a third) order approximation given by the corresponding theorem. The fourth and seventh columns represent the respective relative errors $\left|R_{N}(x) / L(x)\right|$. The fifth and last columns represent the respective relative error bounds given by the corresponding theorem or proposition.

TABle 1. Approximation supplied by (2.2) and error bounds given by (2.4) for $f(t)=e^{-t}$. In this case $c_{n}=\left|a_{n}\right|=1 / n !(f(t)$ verifies the error test).

\begin{tabular}{|c|c|l|l|r|l|l|l|}
\hline$x$ & $L(x) / x$ & $\begin{array}{l}\text { 2nd order } \\
\text { approx. }\end{array}$ & $\begin{array}{l}\text { Rel. } \\
\text { error }\end{array}$ & $\begin{array}{l}\text { Rel. er. } \\
\text { bound }\end{array}$ & $\begin{array}{l}\text { 3rd order } \\
\text { approx. }\end{array}$ & $\begin{array}{l}\text { Rel. } \\
\text { error }\end{array}$ & $\begin{array}{l}\text { Rel. er. } \\
\text { bound }\end{array}$ \\
\hline 100 & $1.62121 \mathrm{e}-4$ & $1.64493 \mathrm{e}-4$ & 0.0146 & 0.0148 & $1.62089 \mathrm{e}-4$ & $1.977 \mathrm{e}-4$ & $2 . \mathrm{e}-4$ \\
1000 & $1.64253 \mathrm{e}-6$ & $1.64493 \mathrm{e}-6$ & 0.001461 & 0.001464 & $1.64252 \mathrm{e}-6$ & $1.974 \mathrm{e}-6$ & $1.977 \mathrm{e}-6$ \\
10000 & $1.64469 \mathrm{e}-8$ & $1.64493 \mathrm{e}-8$ & $1.4615 \mathrm{e}-4$ & $1.4617 \mathrm{e}-4$ & $1.64469 \mathrm{e}-8$ & $1.9737 \mathrm{e}-8$ & $1.974 \mathrm{e}-8$ \\
\hline
\end{tabular}

TABle 2. Approximation supplied by (2.2) and error bounds given by (2.4) for $f(t)=e^{-t}$ and $\operatorname{Arg}(x)=\pi / 4$.

\begin{tabular}{|c|c|c|c|c|c|c|c|}
\hline$|x|$ & $L(x) / x$ & $\begin{array}{l}\text { 2nd order } \\
\text { approx. }\end{array}$ & $\begin{array}{l}\text { Rel. } \\
\text { error }\end{array}$ & $\begin{array}{l}\text { Rel. er. } \\
\text { bound }\end{array}$ & $\begin{array}{l}\text { 3rd order } \\
\text { approx. }\end{array}$ & $\begin{array}{l}\text { Rel. } \\
\text { error }\end{array}$ & $\begin{array}{l}\text { Rel. er. } \\
\text { bound }\end{array}$ \\
\hline 10 & $\begin{array}{r}0.00140423- \\
0.014774 \mathrm{i}\end{array}$ & $\begin{array}{r}0.00169996- \\
0.01474937 \mathrm{i}\end{array}$ & 0.02 & 0.03 & $\begin{array}{r}0.00137526- \\
0.01474937 \mathrm{i}\end{array}$ & 0.0026 & 0.003 \\
\hline 100 & $\begin{array}{r}1.66779 \mathrm{e}-6- \\
0.0001628 \mathrm{i}\end{array}$ & $\begin{array}{r}1.699965 \mathrm{e}-6- \\
0.00016279 \mathrm{i}\end{array}$ & $1.97 \mathrm{e}-4$ & $1.99 \mathrm{e}-4$ & $\begin{array}{r}1.667495 \mathrm{e}-6- \\
0.00016279 \mathrm{i}\end{array}$ & $2.53 \mathrm{e}-6$ & $2.54 \mathrm{e}-5$ \\
\hline 1000 & $\begin{array}{r}1.69672 \mathrm{e}-9- \\
1.64323 \mathrm{e}-6 \mathrm{i}\end{array}$ & $\begin{array}{r}1.699965 \mathrm{e}-9- \\
1.643234 \mathrm{e}-6 \mathrm{i}\end{array}$ & $1.974 \mathrm{e}-6$ & $1.976 \mathrm{e}-7$ & $\begin{array}{r}1.696718 \mathrm{e}-9- \\
1.643234 \mathrm{e}-6 \mathrm{i}\end{array}$ & $2.522 \mathrm{e}-9$ & $2.524 \mathrm{e}-9$ \\
\hline
\end{tabular}


TABLE 3. Approximation supplied by 2.5 and error bounds given by (2.6) for $f(t)=e^{-t}$ and $\operatorname{Arg}(x)=\pi / 3$.

\begin{tabular}{|c|c|c|c|c|c|c|c|}
\hline$|x|$ & $L(x) / x$ & $\begin{array}{l}\text { 1st order } \\
\text { approx. }\end{array}$ & $\begin{array}{l}\text { Rel. } \\
\text { error }\end{array}$ & $\begin{array}{l}\text { Rel. er. } \\
\text { bound }\end{array}$ & $\begin{array}{l}\text { 2nd order } \\
\text { approx. }\end{array}$ & $\begin{array}{l}\text { Rel. } \\
\text { error }\end{array}$ & $\begin{array}{l}\text { Rel. er. } \\
\text { bound }\end{array}$ \\
\hline 0.005 & $\begin{array}{r}99.5004- \\
173.204 \mathrm{i}\end{array}$ & $\begin{array}{c}100 .- \\
173.205 \mathrm{i}\end{array}$ & 0.002501 & 0.002503 & $\begin{array}{r}99.5- \\
173.205 \mathrm{i}\end{array}$ & $4.17186 \mathrm{e}-6$ & $4.17188 \mathrm{e}-6$ \\
\hline 0.0005 & $\begin{array}{r}999.5- \\
1732.0507 \mathrm{i}\end{array}$ & $\begin{array}{c}1000 .- \\
1732.0508 \mathrm{i}\end{array}$ & $2.5001 \mathrm{e}-4$ & $2.5003 \mathrm{e}-4$ & $\begin{array}{r}999.5- \\
1732.0508 \mathrm{i}\end{array}$ & $4.164 \mathrm{e}-8$ & $4.167 \mathrm{e}-8$ \\
\hline 0.00005 & $\begin{array}{r}9999.5- \\
17320.508 \mathrm{i}\end{array}$ & $\begin{array}{r}10000 .- \\
17320.508 \mathrm{i}\end{array}$ & $2.50001 \mathrm{e}-5$ & $2.50003 \mathrm{e}-4$ & $\begin{array}{c}9999.5- \\
17320.508 \mathrm{i}\end{array}$ & $3.79 \mathrm{e}-10$ & 4.e-10 \\
\hline
\end{tabular}

TABLE 4. Approximation supplied by (2.14) and error bound given by 2.27) for $f(t)=t^{2 / 3} /(1+t)$ and $x>0$. In this case $c_{n}=\left|a_{n}\right|$ $(f(t)$ verifies the error test).

\begin{tabular}{|l|c|c|r|r|r|r|r|}
\hline$x$ & $L(x)$ & $\begin{array}{l}\text { 2nd order } \\
\text { approx. }\end{array}$ & $\begin{array}{l}\text { Rel. } \\
\text { error }\end{array}$ & $\begin{array}{l}\text { Rel. er. } \\
\text { bound }\end{array}$ & $\begin{array}{l}\text { 3rd order } \\
\text { approx. }\end{array}$ & $\begin{array}{l}\text { Rel. } \\
\text { error }\end{array}$ & $\begin{array}{l}\text { Rel. er. } \\
\text { bound }\end{array}$ \\
\hline 0.1 & 6.67715 & 5.27033 & 0.2 & 0.56 & 6.62732 & 0.007 & 0.06 \\
0.01 & 38.3712 & 37.6729 & 0.018 & 0.04 & 38.3688 & $6.25 \mathrm{e}-5$ & $5 . \mathrm{e}-4$ \\
0.001 & 188.402 & 188.072 & 0.0017 & 0.004 & 188.402 & $6 . \mathrm{e}-7$ & $4.8 \mathrm{e}-6$ \\
\hline
\end{tabular}

TABLE 5. Approximation supplied by (2.14) and error bound given by (2.27) for $f(t)=t^{2 / 3} /(1+t)$ and $\operatorname{Arg}(x)=\pi / 4$.

\begin{tabular}{|c|c|c|l|r|r|l|c|}
\hline$|x|$ & $L(x)$ & $\begin{array}{l}\text { 1st order } \\
\text { approx. }\end{array}$ & $\begin{array}{l}\text { Rel. } \\
\text { error }\end{array}$ & $\begin{array}{l}\text { Rel. er. } \\
\text { bound }\end{array}$ & $\begin{array}{l}\text { 2nd order } \\
\text { approx. }\end{array}$ & $\begin{array}{l}\text { Rel. } \\
\text { error }\end{array}$ & $\begin{array}{l}\text { Rel. er. } \\
\text { bound }\end{array}$ \\
\hline 0.05 & $6.90631-$ & $6.42398-$ & 0.065 & 0.18 & $6.90317-$ & $9.7 \mathrm{e}-4$ & $8.9 \mathrm{e}-3$ \\
& $3.78924 \mathrm{i}$ & $3.96219 \mathrm{i}$ & & & $\begin{array}{c}3.79623 \mathrm{i} \\
27.2709-\end{array}$ & $8.5 \mathrm{e}-6$ & $7.4 \mathrm{e}-5$ \\
0.005 & $27.271-$ & $27.1074-$ & 0.0059 & 0.015 & \\
& $12.4648 \mathrm{i}$ & $12.5295 \mathrm{i}$ & & & $\begin{array}{c}12.4651 \mathrm{i} \\
92.567-\end{array}$ & $1.68 \mathrm{e}-7$ & $6.996 \mathrm{e}-7$ \\
0.0005 & $92.567-$ & $92.5141-$ & $5.68 \mathrm{e}-4$ & 0.001 & $39.6003 \mathrm{i}$ & & \\
& $39.6003 \mathrm{i}$ & $39.6219 \mathrm{i}$ & & & & & \\
\hline
\end{tabular}


TABLE 6. Approximation supplied by (2.19) and error bound given by $\operatorname{Min}\{(2.29),(2.30)\}$ for $f(t)=1 /(1+t)$ and $x>0$. In this case $c_{n}=\left|a_{n}\right|(f(t)$ verifies the error test. $)$

\begin{tabular}{|l|c|l|r|r|r|l|r|}
\hline$x$ & $L(x)$ & $\begin{array}{l}\text { 1st order } \\
\text { approx. }\end{array}$ & $\begin{array}{l}\text { Rel. } \\
\text { error }\end{array}$ & $\begin{array}{l}\text { Rel. er. } \\
\text { bound }\end{array}$ & $\begin{array}{l}\text { 2nd order } \\
\text { approx. }\end{array}$ & $\begin{array}{l}\text { Rel. } \\
\text { error }\end{array}$ & $\begin{array}{l}\text { Rel. er. } \\
\text { bound }\end{array}$ \\
\hline 0.1 & 2.48469 & 2.30259 & 0.07 & 0.3 & 2.48075 & 0.0016 & 0.03 \\
0.01 & 4.63456 & 4.60517 & 0.006 & 0.02 & 4.6345 & $1.26 \mathrm{e}-5$ & $2 . \mathrm{e}-4$ \\
0.001 & 6.91184 & 6.90776 & $5.9 \mathrm{e}-4$ & $1.97 \mathrm{e}-3$ & 6.91184 & $1 . \mathrm{e}-7$ & $1.7 \mathrm{e}-6$ \\
\hline
\end{tabular}

TABLE 7. Approximation supplied by (2.19) and error bound given by $\operatorname{Min}\{2.29), 2.30\}$ for $f(t)=1 /(1+t)$ and for $\operatorname{Arg}(x)=\pi / 5$.

\begin{tabular}{|c|c|c|c|c|c|c|c|}
\hline$|x|$ & $L(x)$ & $\begin{array}{l}\text { 1st order } \\
\text { approx. }\end{array}$ & $\begin{array}{l}\text { Rel. } \\
\text { error }\end{array}$ & $\begin{array}{l}\text { Rel. er. } \\
\text { bound }\end{array}$ & $\begin{array}{l}\text { 2nd order } \\
\text { approx. }\end{array}$ & $\begin{array}{l}\text { Rel. } \\
\text { error }\end{array}$ & $\begin{array}{l}\text { Rel. er. } \\
\text { bound }\end{array}$ \\
\hline 0.05 & $\begin{array}{l}3.09152- \\
0.577448 \mathrm{i}\end{array}$ & $\begin{array}{c}2.99573- \\
0.628319 \mathrm{i}\end{array}$ & 0.03 & 0.1 & $\begin{array}{l}3.09105- \\
0.57848 \mathrm{i}\end{array}$ & $3.6 \mathrm{e}-4$ & $6.4 \mathrm{e}-3$ \\
\hline 0.005 & $\begin{array}{l}5.31251- \\
0.619936 \mathrm{i}\end{array}$ & $\begin{array}{r}5.29832- \\
0.62832 \mathrm{i}\end{array}$ & 0.003 & 0.01 & $\begin{array}{r}5.31251- \\
0.61995 \mathrm{i}\end{array}$ & 3.e-6 & $4.8 \mathrm{e}-5$ \\
\hline 0.0005 & $\begin{array}{r}7.60279- \\
0.627143 \mathrm{i}\end{array}$ & $\begin{array}{r}7.6009- \\
0.628319 \mathrm{i}\end{array}$ & $2.9 \mathrm{e}-4$ & $9.5 \mathrm{e}-4$ & $\begin{array}{l}7.60279- \\
0.627143 \mathrm{i}\end{array}$ & $2.7 \mathrm{e}-8$ & 4.e-7 \\
\hline
\end{tabular}

4. Acknowledgments. The financial support of The Government of Navarra, Res. 134/2002, The Government of Aragón, Cod. 245/69 and DGCYT (BFM2000-0803) is acknowledged.

\section{REFERENCES}

[1] M. Abramowitz and I.A. Stegun, Handbook of mathematical functions with formulas, graphs, and mathematical tables, Dover, New York, 1966. MR0208797 (34:8606)

[2] C. Ferreira and J. L. López, An Asymptotic Expansion of the Double Gamma Function, J. of Approx. Theory. vol. 111, 2001, pp. 298-314. MR.1849551 (2002g:41049)

[3] C. Ferreira and J. L. López, Asymptotic expansions of generalized Stieltjes transforms of algebraically decaying functions, Stud. Appl. Math. vol. 108, 2002, pp. 187-215. MR1883093 (2003c:44007)

[4] R. R. Goldberg, Inversions of generalized Lambert transforms, Duke Math. J. vol. 25, 1958, pp. 459-476. MR0095390 (20:1893)

[5] S. P. Goyal and R. K. Laddha, On the generalized Riemann zeta functions and the generalized Lambert transform, Gadnita Sandesh. vol. 11, n. 2, 1997, pp. 99-108. MR.1641564 (2000b:11106)

[6] N. Hayek, B. J. González and E. R. Negrin, The generalized Lambert transform on distributions of compact support, J. Math. Anal. Appl. vol. 275, 2002, pp. 938-944. MR1943789 (2003k:46051)

[7] E. L. Miller, Summability of power series using the Lambert transform, Rev. Univ. Nac. Tucumán, Series A vol. 28, n. 1,2, 1978, pp. 89-106. MR0839840 (87g:40003)

[8] E. L. Miller, Convergence properties and an inversion formula for the Lambert transform, Rev. Tech. Ingr. Univ. Zulia vol. 2, n. 1,2, 1979, pp. 77-94. MR0549310(80j:44006) 
[9] E. R. Negrín, The Lambert transform on $\epsilon^{\prime}$, Portugal Math, vol. 50, n. 3, 1993, pp. 359-363. MR.1244514 (94i:44004)

[10] F.W.J. Olver, Special Functions: Asymptotics and Special Functions, Academic Press, New York, 1974. MR0435697(55:8655)

[11] W. B. Pennington, Widder's inversion formula for the Lambert transform, Duke Math., vol. 27, 1960, pp. 561-568. MR0116187 (22:6982)

[12] R. K. Raina and T. S. Nahar, A note on a certain class of functions related to Hurwitz zeta function and Lambert transform, Tamkang J. Math, vol. 31, n.1, 2000, pp. 49-56. MR.1745770|(2000m:11079)

[13] R. K. Raina and H. M. Srivastava, Certain results associated with the generalized Riemann zeta functions, Rev. Tecn. Fac. Ingr. Univ. Zulia, vol. 18, n.3, 1995, pp. 301-304. MR.1372687 (96m:11074)

[14] R.E.A.C. Paley and N. Wiener, Fourier transforms in the complex domain, Reprint of the 1934 original. Amer. Math. Soc. Coll. Publ., vol. 19, 1987. MR1451142 (98a:01023)

[15] J. Rodero Carrasco, The Lambert transform and an inversion formula, Rev. Acad. Ci. Madrid, vol. 53, 1959, pp. 727-736. MR0125417 (23:A2720)

[16] D. V. Widder, An inversion of the Lambert transform, Math. Mag, vol. 23, 1950, pp. 171-182. MR0036864 (12:175c)

[17] R. Wong, Asymptotic Approximations of Integrals, Academic Press, Boston, 1989. MR1016818 (90j:41061)

[18] A. I. Zayed, Handbook of Function and Generalized Function Transformations, CRC Press, New York, 1996. MR1392476 (97h:44001) 